\title{
Inverse Consistent Mapping in 3D Deformable Image Registration: Its Construction and Statistical Properties
}

\author{
Alex Leow ${ }^{1}$, Sung-Cheng Huang ${ }^{2}$, Alex Geng ${ }^{1}$, James Becker ${ }^{3}$, Simon Davis ${ }^{3}$, Ar- \\ thur Toga ${ }^{1}$, and Paul Thompson ${ }^{1}$
}

${ }^{1}$ Rm. 4238, 710 Westwood Plaza, LONI, UCLA School of Medicine

${ }^{2}$ Dept. of Molecular and Medical Pharmacology, UCLA School of Medicine

${ }^{3}$ Dept. of Neurology, Psychiatry, and Psychology, Univ. of Pittsburgh

aliao@loni.ucla.edu

\begin{abstract}
This paper presents a new approach to inverse consistent image registration. A uni-directional algorithm is developed using symmetric cost functionals and regularizers. Instead of enforcing inverse consistency using an additional penalty that penalizes inconsistency error, the new algorithm directly models the backward mapping by inverting the forward mapping. The resulting minimization problem can then be solved uni-directionally involving only the forward mapping, without optimizing in the backward direction. Lastly, we evaluated the algorithm by applying it to the serial MRI scans of a clinical case of semantic dementia. The statistical distributions of the local volume change (Jacobian) maps were examined by considering the Kullback-Liebler distances on the material density functions. Contrary to common belief, the values of any non-trivial Jacobian map do not follow a log-normal distribution with zero mean. Statistically significant differences were detected between consistent versus inconsistent matching when permutation tests were performed on the resulting deformation maps
\end{abstract}

\section{Introduction}

Non-linear image registration is a well-established field in medical imaging with many applications in functional and anatomic brain mapping, image guided surgery, and multimodality image fusion [1-3]. The goal of image registration is to align, or spatially normalize, one image to another. In multisubject studies, registration reduces subject-specific anatomic differences by deforming individual images onto a population average brain template. Using a similar procedure, maps visualizing structural brain change over time can be generated by deforming baseline scans onto subsequent scans of the same subject, and using the deformation map to quantify local changes. To formulate the image registration problem mathematically, we denote the two images to be registered as $T$ and $S$ (both defined on an image domain $\Omega$ ). We seek to estimate a transformation $h$ so that $S(h(x))$ is "closest" to $T(x)$ in terms of certain matching criteria. Ideally, this transformation mapping $h$ should be smooth, one-toone, and differentiable (i.e., a diffeomorphism). Conventionally, researchers in the field of non-linear image registration use the notation $u=\left(u_{x}, u_{y}, u_{z}\right)$, the displacement 
vector field away from the identity map, to represent the transformation $h$ (i.e., $h(x)=x-u(x))$. The inverse map $h^{-1}$ of $h$ (i.e., $h^{-1}(h(x))=x$ for all $x$ ) thus maps the target to the source image. We will also use the notation $u^{-1}$ to denote the displacement field of the inverse map $h^{-1}$.

To make the transformation smooth, one-to-one, and differentiable, a regularizing constraint on the displacement field is needed. Thus, the problem of image registration is often cast as a minimization problem with a total cost functional $E$ expressed in general as $E=E_{M}(S, T)+R(h)$, where $E_{M}$ is the matching criterion cost function, and $R(h)$ is the regularizing constraint on the transformation.

Intuitively, the problem of image registration is symmetric, i.e., the correspondences established between the two images should not depend on the order we use to compare the two images. However, early approaches for non-linear image registration were not symmetric and various terms (e.g., source, target, template, study, and reference) have been used to describe the direction of this comparison. In this paper, we will adopt the term source or S to describe the floating/deforming image and the term target or $\mathrm{T}$ to describe the image that the source image is deformed to match. This dependence on the direction of comparison not only complicates the notation but also has serious disadvantages. Firstly, the deformation field depends on which image is assigned the source and which image the target. This dependence can be termed inverse inconsistency as inconsistency arises if we switch the order of source and target. Secondly, as pointed out in [4], these inversely inconsistent approaches penalize the expansion of image regions more than the shrinkage of image regions. This imbalance in the penalty was also noticed and discussed in another paper [5] by the same group in which shrinking brain lesions were found to be easier to detect than expanding ones using inversely inconsistent methods. Thus, conventional inverse-inconsistent nonlinear registration techniques may be problematic in applications where the Jacobian of the transformation $h$ is interpreted as measuring tissue loss or expansion, a step commonly performed in computational neuroanatomy (e.g., in tensor-based morphometry).

One of the first approaches for inverse consistent registration [6] symmetrized not only the matching cost functional, but also the regularization of the displacement. Using the sum of squared differences of the intensities as the matching cost functional, the following total cost function $E$ was proposed:

$$
E(T, S)=\underbrace{\int_{\Omega}|S(h(x))-T(x)|^{2} d x+\lambda R(h)}_{E_{1}}+\underbrace{\int_{\Omega}\left|T\left(h^{-1}(x)\right)-S(x)\right|^{2} d x+\lambda R\left(h^{-1}\right)}_{E_{2}}
$$

Here $\lambda$ is a positive scalar weighting of the regularizers applied to the forward and inverse mappings. The above cost function is symmetric and does not depend on the order of $T$ and $S$, i.e., $E(T, S)=E(S, T)$. To solve (1) numerically, [6] solved for $h$ and $g$ separately as follows and additional inverse consistency constraints were added so that $g$ numerically realized $h^{-1}$. 


$$
\begin{aligned}
& E_{h}(T, S)=\int_{\Omega}|S(h(x))-T(x)|^{2} d x+\lambda R(h)+\rho \int_{\Omega}\left\|h-(g)^{-1}\right\|^{2} d x \\
& E_{g}(T, S)=\int_{\Omega}|T(g(x))-S(x)|^{2} d x+\lambda R(g)+\rho \int_{\Omega}\left\|g-(h)^{-1}\right\|^{2} d x .
\end{aligned}
$$

Iterative gradient descent methods can be employed and the numerical algorithm for minimizing eq. (2) can be summarized as follows. At initialization, both $h$ and $g$ are set to be the identity map. At each time step, the gradient descent of $E_{h}$ is computed to update $h$ while fixing the map $g$, and similarly $E g$ is used to update $g$ while fixing the map $h$. This avoids the highly nonlinear nature of the original minimization problem eq. (1) in which both the forward and backward mappings are involved and need to be optimized while maintaining the inverse relation between them.

Although the alternative formulation eq. (2) was extensively tested, with good experimental results, it has some disadvantages compared to the original formulation in eq. (1). Firstly, the algorithm proposed to solve eq. (2) is essentially a two step strategy and creates a lagging-behind situation in estimating $h$ and $g$. Either $h$ and $g$ has to be alternately fixed (i.e., the two maps are not estimated simultaneously). Moreover, an extra weighting parameter for the inverse consistency constraints has to be considered and was tuned case-by-case in [6].

\section{Method}

\subsection{Inverting gradient descent direction}

We seek to solve the original symmetric formulation for non-linear registration in eq. (1) instead of the modified formulation in eq. (2). To this end, we propose to directly couple the backward and forward mappings, allowing all driving body forces to be combined in the forward direction. As a result, the corresponding minimization problem can be optimized in a unidirectional fashion, i.e. by considering the forward mapping only. Thus, the proposed algorithm can be thought of as a unidirectional procedure with embedded inverse consistency.

To simplify the derivation of this procedure, we will illustrate it using the sum of squared difference (SSD) as the matching cost functional. This can easily be extended to other intensity/feature-based cost functionals. As mentioned before, we will convert the gradient descent direction involving the backward mapping $\left(E_{2}\right.$ in eq. (1)) to a corresponding gradient descent direction in the forward direction. More precisely, we wish to update $h$ and $h^{-1}$ by perturbing the mappings from the previous time step in a descent direction with respect to the total cost functional $E$.

$$
h \rightarrow h+\varepsilon \eta_{1}+\varepsilon \eta_{2} ; h^{-1} \rightarrow h^{-1}+\varepsilon \xi_{1}+\varepsilon \xi_{2} .
$$

Here, $\varepsilon$ is an infinitesimally small positive number and $\eta_{1}$ and $\xi_{1}$ are vector fields that represent the gradient descent direction of $E_{l}$ in eq. (1) in the forward and back- 
ward direction respectively, with $\eta_{2}$ and $\xi_{2}$ similarly defined for the term $E_{2}$. Notice that the terms $\eta_{1}$ and $\xi_{2}$ can be computed using standard calculus. Formally, we can write $\eta_{1}$ and $\xi_{2}$ as follows

$$
\begin{aligned}
& \eta_{1}(x)=(S(h(x))-T(x)) \nabla S(h(x))+\lambda \nabla R(h) ; \\
& \xi_{2}(x)=\left(T\left(h^{-1}(x)\right)-S(x)\right) \nabla T\left(h^{-1}(x)\right)+\lambda \nabla R\left(h^{-1}\right) .
\end{aligned}
$$

Here the gradient operator applied to the regularizer denotes the gradient descent direction of the regularizer (or a regularized/smoothed body force). In order to numerically compute (3), we need to solve for $\eta_{2}$ and $\xi_{1}$ using eqs. (3) and (4). To this end, we first utilize the inverse relationship given in eq. (3)

$$
\left(h^{-1}+\varepsilon \xi_{1}+\varepsilon \xi_{2}\right) \circ\left(h+\varepsilon \eta_{1}+\varepsilon \eta_{2}\right)=i d .
$$

where $i d$ is the identity mapping. By expanding (5) using Taylor's expansion and collecting up to first order terms of $\varepsilon$, we obtain

$$
D h^{-1}(h(x))\left\{\eta_{1}(x)+\eta_{2}(x)\right\}=-\xi_{1}(h(x))-\xi_{2}(h(x)) .
$$

Here $D$ denotes the Jacobian matrix operator. Using the relationship: $(D(h(x)))^{-1}=D h^{-1}(h(x))$, derived by differentiating the identity relation $h^{-1}(h)=i d$, we obtain the following alternative form to (6)

$$
\eta_{1}(x)+\eta_{2}(x)=-D(h(x))\left\{\xi_{1}(h(x))+\xi_{2}(h(x))\right\} .
$$

With (7), we can now express $\eta_{2}$ and $\xi_{1}$ using the known quantities $\xi_{2}$ and $\eta_{1}$

$$
\begin{aligned}
& \eta_{2}(x)=-D(h(x)) \xi_{2}(h(x)) ; \\
& \xi_{1}(x)=-D\left(h^{-1}(x)\right) \eta_{1}\left(h^{-1}(x)\right)
\end{aligned}
$$

With all the quantities known, we now have a recipe for minimizing the symmetric forward-backward problem (1) using an iterative approach with the updating formulae (3). Moreover, as the two updating formulae in (3) are designed to be consistent with each other, we can simply update in the forward direction (first formula) without using the backward updating formulae at all. Notice that with eq. (8), the inversion of a body force from the backward direction to the forward direction can be carried out using only the forward mapping $h$ (without involving $h^{-1}$ ). This property is desirable due to the unavoidable numerical errors incurred when inverting between $h$ and $h^{-1}$.

Thus, at each time step of the gradient descent method, we sum up the total forward body force by combining the forward body force and the inverted backward body force obtained by applying (8). To evaluate (8) numerically, interpolations are necessary and we use a bi-linear or tri-linear technique to interpolate the backward body force in the non-grid point position $h(x)$.

In this paper, the linear elastic operator is chosen as in [6] for the regularizer 


$$
R(u)=\int\|-\alpha \Delta u-\beta \nabla(\nabla \cdot u)\|^{2} d x
$$

where $\Delta$ is the Laplacian and $\alpha$ and $\beta$ are the Lamé constants (both set to be 1.0 ). The Fast Fourier transform technique (FFT) is applied to parameterize the displacement field. A multi-resolution minimization scheme can then be implemented in the frequency domain.

\subsection{Statistical properties of deformation maps}

In this section, we discuss the statistical properties of the deformation maps arising from non-linear image registration. In tensor-based morphometry, the Jacobian determinants of deformation maps are used to index local volume compressions or dilations and their distribution is typically assumed to be log-normal [7]. However, we now show that the determinant of the Jacobian operator applied to any bijective (oneto-one and onto) globally volume-preserving mapping $h$ cannot have a log-normal distribution with zero mean. To this end, let us denote the Jacobian matrix of a transformation $h$ as $D h$ (with the (i,j)-th element $\partial h_{j} / \partial x_{i}$ ), and the local volume loss/expansion map (Jacobian map) can thus be defined as $J(x)=|D h(x)|$. Notice that $J$ encodes the local volume change of the source with respect to the target image, and may be considered to reside on the target reference frame. Since $h$ is a diffeomorphic and bijective mapping from $\Omega$ to itself, we obtain the following using a change of variable

$$
\frac{1}{|\Omega|} \int_{\Omega}|D h(x)| d x \stackrel{y=h(x)}{=} \frac{1}{|\Omega|} \int_{\Omega} d y=1 .
$$

Here, the first integral should be evaluated with respect to the target domain and the second integral with respect to the source domain, and $|\Omega|$ is the total volume of $\Omega$. Given eq. (10), we can define a probability density function (PDF) $P$ on $\Omega$ as $P(x)=|D h(x)| /|\Omega|$ as it integrates to 1 . Let us also use $Q(x)=1 /|\Omega|$ to denote the PDF of the uniform distribution on $\Omega$. Then, using the relation $\log (1 / a)=-\log (a)$, we can compute the mean of $\log (J)$ in the target reference frame on $\Omega$ as follows

$$
\begin{aligned}
& \frac{1}{|\Omega|} \int_{\Omega} \log |\operatorname{Dh}(x)| d x=-\int_{\Omega} \frac{1}{|\Omega|} \log \left(\frac{1 /|\Omega|}{|D h(x)| /|\Omega|}\right) d x \\
& =-\int_{\Omega} Q \log \frac{Q}{P} d x=-K L(Q, P) .
\end{aligned}
$$

Here $K L$, the non-negative asymmetric Kullback-Leibler $(K-L)$ distance, between two PDF's $\mathrm{X}$ and $\mathrm{Y}$ is defined as 


$$
\begin{aligned}
& K L(X, Y)=E_{X}\left[\frac{X}{Y}\right]=\int_{\Omega} X \log \frac{X}{Y} d x \geq 0 ; \\
& K L(X, Y) \neq K L(Y, X) ; K L(X, Y)=0 \text { iff } X \equiv Y .
\end{aligned}
$$

Eq. (12) suggests that calculating the mean of a log-transformed volume change map is the same as computing the negative $K-L$ distance between $Q$ and $P$, and is always non-positive (zero only when $J$ equals 1 everywhere, i.e., when the flow is incompressible). By contrast, let us also show that the log transform of $J \circ h^{-1}=J\left(h^{-1}(x)\right)$, the volume change map pulled back onto the source reference frame, has a mean larger than zero, unless the flow is incompressible. Thus, the pulled-back Jacobian map does not have a log-normal distribution with zero mean either.

$$
\begin{aligned}
& \frac{1}{|\Omega|} \int_{\Omega} \log J\left(h^{-1}(y)\right) d y \stackrel{y=h(x)}{=} \frac{1}{|\Omega|} \int_{\Omega}(\log J(x))|D h| d x \\
= & \frac{1}{|\Omega|} \int_{\Omega}(\log |D h(x)|)|D h| d x=\int_{\Omega} \frac{|D h|}{|\Omega|} \log \left(\frac{|D h(x)| / \Omega \mid}{1 /|\Omega|}\right) d x \\
= & \int_{\Omega} P \log \frac{P}{Q} d x=K L(P, Q) .
\end{aligned}
$$

Similarly, calculating the mean of the pulled-back logged volume change map is equivalent to computing the $K-L$ distance between $\mathrm{P}$, and $\mathrm{Q}$, and is always nonnegative. Conventional log-normal modeling of the Jacobian distributions may therefore be less appropriate than non-parametric estimation, as illustrated in the Results.

\section{Results}

3D T1-weighted magnetic resonance images (MRI) of a 57 year-old male patient diagnosed with semantic dementia were obtained using a gradient echo acquisition (TR $25 \mathrm{~ms}$, TE $5 \mathrm{~ms}$, slice thickness $1.5 \mathrm{~mm}$, FOV $24 \times 18 \mathrm{~cm}$, flip angle 40 degrees, no gaps). A total of four serial scans were obtained (baseline scan in 02/1993; follow-up scans in 10/1994, 02/1996, and 08/1999). The baseline (target) and the final followup (source) scans were used to evaluate the proposed approach. The two scans were first rigidly aligned and re-sliced to an isotropic volume of size $180 \times 180 \times 180$ (a voxel $=1 \mathrm{~mm}^{3}$ ). The proposed inverse consistent registration algorithm was used to deform the source back to the target by maximizing the mutual information (MI) [8] between the deforming source and target images. This spatial normalization of scans over time allowed local tissue change to be estimated as mentioned in previous sections. A multi-resolution scheme starting from the $32 \times 32 \times 32$ FFT resolution was used $(\lambda=1 \mathrm{e}-4$; time step=3e-6), and numerical convergence was checked every 20 iterations (convergence criteria was met when the MI failed to increase by 0.001 after one iteration). 40 iterations were computed in each FFT resolution before the resolu- 
tion was increased by a factor of 2 (with the time step decreased to one-tenth) in each dimension. The top panel of Fig. 1 plots the target image (baseline scan) from an angle showing temporal lobes bilaterally, the second panel the source image from the same angle. The MRI scans show existing left temporal lobe atrophy (LT) with relative preservation of the right temporal lobe (RT). However, closer inspection of the Jacobian map (Fig. 2) shows active atrophy in the right temporal lobe, as well as bilateral caudate (RC, LC), putamen (RP, LP), and thalamus (RT, LT) tissue loss, while no active atrophy was detected in the left temporal lobe during the same time period (not shown here). Fig. 3 plots the values of MI and regularizer versus iterations in the forward and backward direction using (1) the proposed inverse consistent approach, and (2) an inconsistent approach (minimizing only the term $\mathrm{E}_{1}$ in eq. (1)). The proposed consistent algorithm achieved not only higher MI values, but also lower regularizer values.

In order to validate the inverse consistency property of the proposed algorithm, we compared the deformation with that obtained by switching the source/target. Ideally, the deformation should not depend on this order, and thus inverse consistency can be assessed by looking at the difference (Table 1) between the deformation pair. For comparison, the corresponding errors using the inconsistent algorithm are also reported. Notice that the proposed algorithm yielded smaller errors in all aspects, and on average decreased the mean error to about one-seventh compared to the inconsistent algorithm.

We then examined the statistical properties of the $\log (\mathrm{J})$ values. The left panel in the first row of Fig. 4 shows the histogram of the $\log (\mathrm{J})$ values under the proposed inverse consistent mapping (mean -0.0011 ; skewness -0.01657), and the right panel the corresponding histogram using inconsistent matching (mean -0.0017 ; skewness 0.648 ). Notice the slight visual difference in these two histograms. We first tested if the $\log (\mathrm{J})$ distribution is symmetric around mean zero. To this end, a permutation test was performed where 10,000 samples were generated by randomly flipping the sign of each element in the observed distribution (under the null hypothesis that the observed is symmetric around zero). The test statistic was the mean value of the resampled distribution. No re-sampled test statistic (maximum 7.67e-5; minimum $8.23 \mathrm{e}-5$ ) was as extreme as the observed statistic, and thus the null hypothesis was rejected with statistical significance. We then relaxed the null hypothesis and tested the symmetry of $\log (\mathrm{J})$ distribution using another permutation test (random flipping around the observed mean -0.0011$)$ with skewness as the test statistic. The left panel in the second row of Fig. 4 shows the histogram of the re-sampled statistics. The onesided p-value is 0.067 , and thus the null hypothesis (symmetric around its negative mean) cannot be rejected at the 5\% significance level. By contrast, a similar skewness permutation test performed on the $\log (\mathrm{J})$ values under the inconsistent mapping yielded a $\mathrm{p}<0.0001$. Thus, a statistically significant skewness was detected in the case of inconsistent mapping compared to its inverse consistent counterpart. 


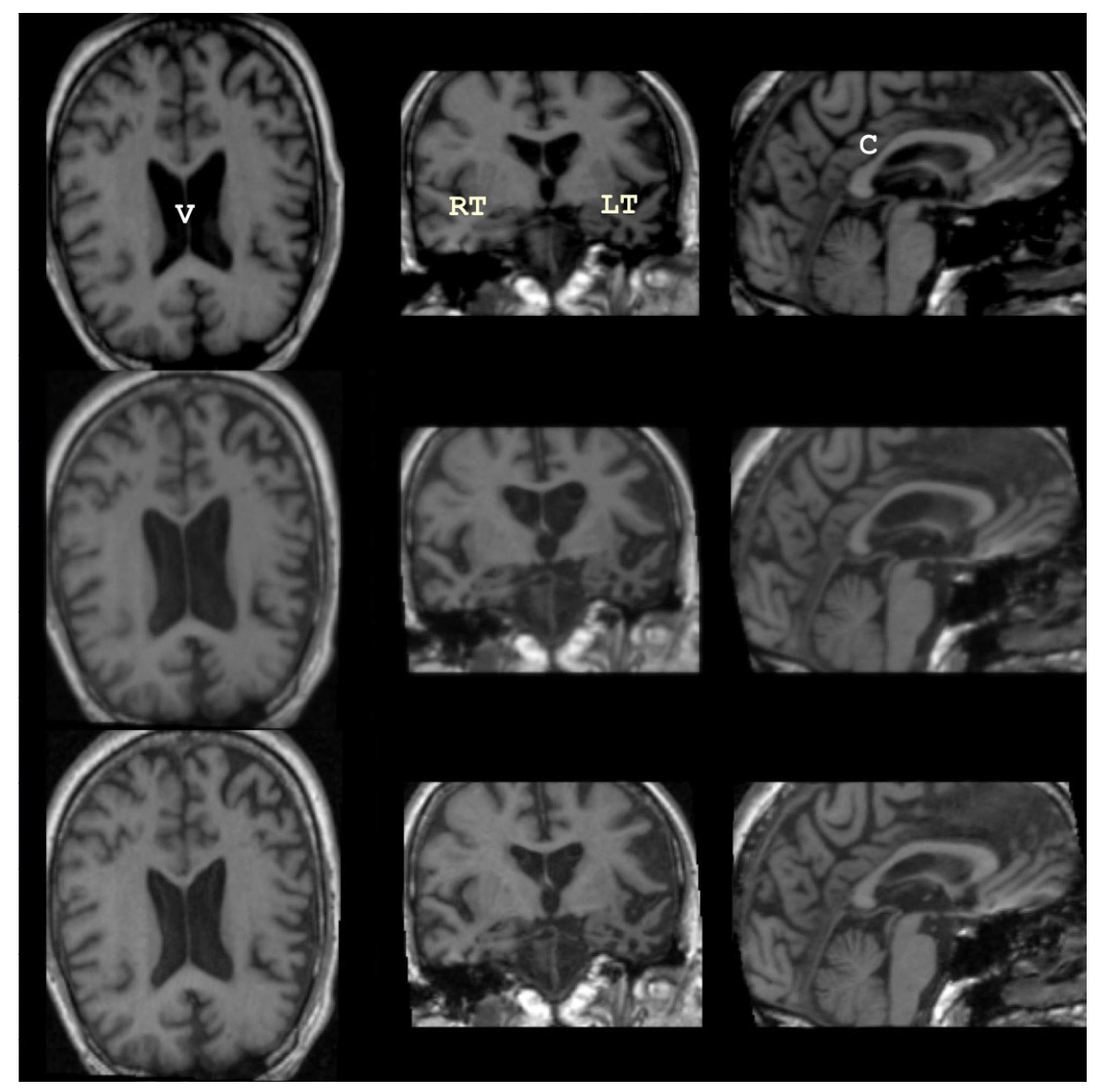

Fig. 1. The first row shows the baseline MRI scan of a patient diagnosed with semantic dementia. The second row shows the follow-up MRI scan of the same patient in which ventricle (V) dilation and copus callosum (C) shape change can be observed. The third row shows the follow-up MRI scan deformed to match the baseline scan using the proposed inverse consistency algorithm with maximization of mutual information (see text).

Finally, we examined the differences in the $\log (\mathrm{J})$ distributions obtained from inverse consistent versus inconsistent mappings. As discussed in previous sections, one would argue that, by equally penalizing positive and negative $\log (\mathrm{J})$ values, an inverse consistent mapping would shift the mean $\log (\mathrm{J})$ value rightward (less negative). We formally tested the statistical significance of this shift using a third permutation test (right panel in the second row of Fig. 4). The test statistic in this case was the difference of the mean $\log (\mathrm{J})$ values between consistent and inconsistent mappings with the observed statistic 6.066e-4. 10,000 samples of this test statistic were calculated by generating two re-sampled distributions using random shuffling of each element in the two observed distributions (under the null hypothesis that the two distributions are identical and share the same mean). Again, not a single re-sampled 
test statistic ( $\max 9.44 \mathrm{e}-5, \min -8.73 \mathrm{e}-5$ ) was as extreme as the observed, and thus a statistically significant difference was detected between the two observed distributions.

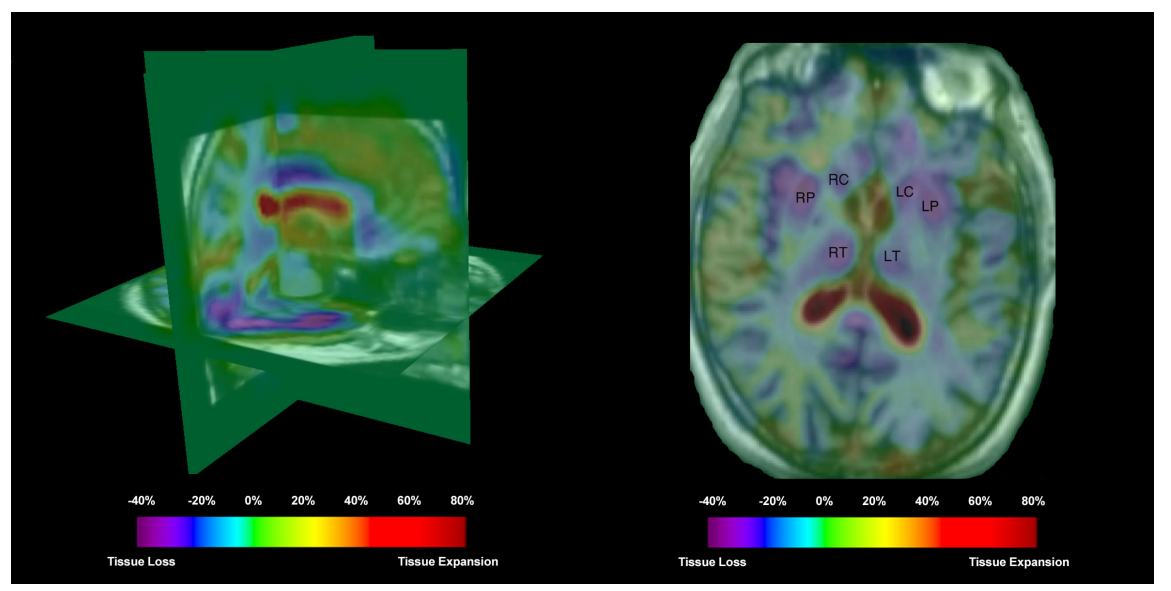

Fig. 2. 3D Jacobian map of the semantic dementia patient shows the active right temporal lobe atrophy (left panel), and deep nuclei involvement (right panel; see text).

Table 1. Statistics of inverse consistency error. * denotes the displacement/deformation obtained by switching the order of the source/target. The numbers are reported with respect to the $64 \times 64 \times 64$ resolution of the FFT parameterization of the displacement.

\begin{tabular}{|c|c|c|c|c|}
\hline \multicolumn{5}{|c|}{ Forward mapping (inverse consistent) } \\
\hline & $\mathrm{u}_{\mathrm{x}}-\mathrm{u}_{\mathrm{x}} *$ & $\mathrm{u}_{\mathrm{y}}-\mathrm{u}_{\mathrm{y}} *$ & $\mathrm{u}_{\mathrm{z}}-\mathrm{u}_{\mathrm{z}}^{*}$ & $\mathrm{~h}-\mathrm{h} *$ \\
\hline Maximum & 0.3893 & 0.8290 & 0.4345 & 0.8616 \\
\hline Mean & 0.0047 & 0.0071 & 0.0049 & 0.0115 \\
\hline \multicolumn{5}{|c|}{ Backward mapping (inverse consistent) } \\
\hline & $\mathrm{u}_{\mathrm{x}}^{-1}-\mathrm{u}_{\mathrm{x}}^{-1} * \mid$ & $\mathrm{u}_{\mathrm{y}}^{-1}-\mathrm{u}_{\mathrm{y}}^{-1} *$ & $\mathrm{u}_{\mathrm{z}}^{-1}-\mathrm{u}_{\mathrm{z}}^{-1} *$ & $\mathrm{~h}^{-1}-\mathrm{h}^{-1} *$ \\
\hline Maximum & 0.2751 & 0.8009 & 0.4145 & 0.8107 \\
\hline Mean & 0.0048 & 0.0071 & 0.0047 & 0.0115 \\
\hline \multicolumn{5}{|c|}{ Forward mapping (inverse inconsistent) } \\
\hline & $\mathrm{u}_{\mathrm{x}}-\mathrm{u}_{\mathrm{x}}^{*}$ & $\mathrm{u}_{\mathrm{y}}-\mathrm{u}_{\mathrm{y}}^{*}$ & $\mathrm{u}_{\mathrm{z}}-\mathrm{u}_{\mathrm{z}}^{*}$ & h-h* \\
\hline Maximum & 0.8343 & 0.8894 & 0.9616 & 0.9617 \\
\hline Mean & 0.0323 & 0.0297 & 0.0360 & 0.0685 \\
\hline \multicolumn{5}{|c|}{ Backward mapping (inverse inconsistent) } \\
\hline & $\left|\mathrm{u}_{\mathrm{x}}^{-1}-\mathrm{u}_{\mathrm{x}}^{-1} *\right|$ & $\mathrm{u}_{\mathrm{y}}^{-1}-\mathrm{u}_{\mathrm{y}}^{-1} *$ & $\mathrm{u}_{\mathrm{z}}^{-1}-\mathrm{u}_{\mathrm{z}}^{-1} *$ & $\mathrm{~h}^{-1}-\mathrm{h}^{-1} *$ \\
\hline Maximum & 0.8499 & 0.9009 & 0.9884 & 0.9579 \\
\hline Mean & 0.0322 & 0.0288 & 0.0362 & 0.0674 \\
\hline
\end{tabular}



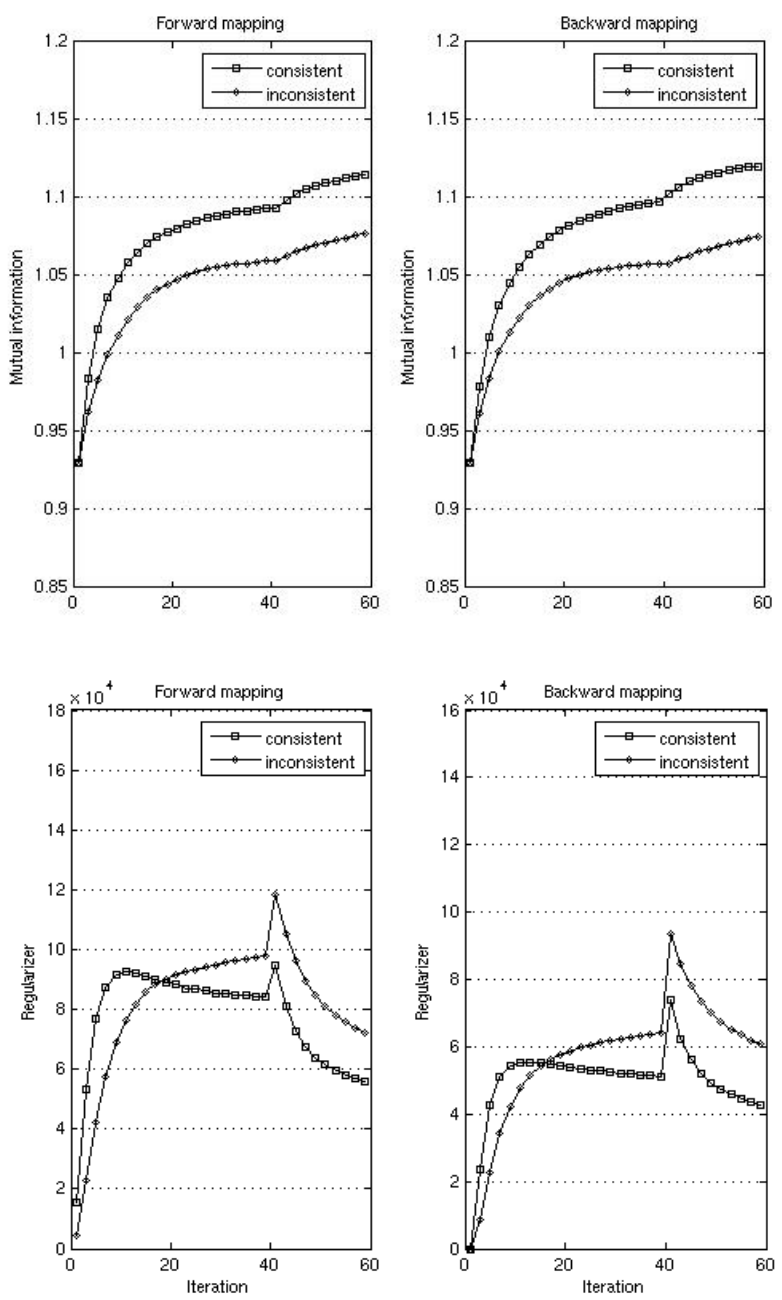

Fig. 3. The Mutual Information (the first row) and the regularizer (the second row) are plotted against the iteration number (x axis) in both the forward (left panel) and backward (right panel) direction. The transient increase of the values around iteration 40 is due to the upsampling of the displacement FFT parameterization. 

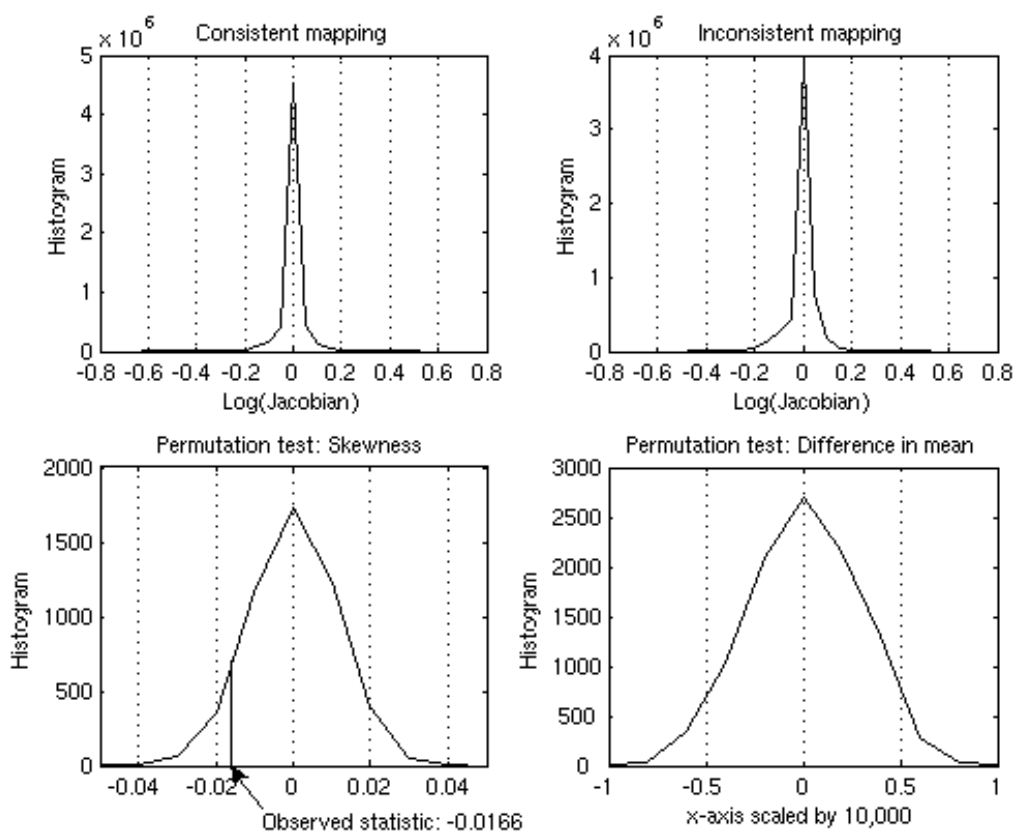

Fig. 4. Skewness of Logged Jacobian distributions. The first row shows the histogram of the $\log (\mathrm{J})$ values of the inverse consistent mapping constructed using the proposed algorithm (left panel), and the corresponding histogram using the inconsistent matching (right panel). Second Row: A permutation test is performed to determine if the consistent matching yields $\log (\mathrm{J})$ values symmetric around its mean (left panel shows the histogram of the re-sampled skewness statistic) with a one-sided $p$-value of 0.067 . Another permutation test is performed to determine if the two distributions in the first row are statistically different. The right panel shows the histogram of the re-sampled test statistic (the values scaled by $10^{4}$ ) where no re-sampled statistic is as extreme as the observed (i.e., $\mathrm{p}<0.0001$ ).

\section{Conclusion}

In this paper, we developed an inverse consistent image registration approach by applying variation calculus principles to the forward mapping only. We characterized the statistical properties of the Jacobian maps, both empirically and by applying the Kullback-Liebler distance to the set of material density functions in both target and source coordinates. We showed that the mean value of any log Jacobian map is always negative except for the trivial case where the Jacobian map is identically one on the whole image domain (incompressible flow). By contrast, any non-trivial log Jacobian map pulled back to the source coordinate must have a positive mean value. Thus, contrary to common belief, the values of any non-trivial Jacobian map do not follow a log normal distribution with zero mean. We also showed that compared to inconsistent matching, consistent matching reduces the skewness and increases the mean value of the $\log (\mathrm{J})$ distribution (making it more symmetric and thus allowing more 
unbiased detection of expanding and shrinking regions). Moreover, the statistical theory of these distributions has strong ties with formulations in information theory. Our conclusion has important consequences when performing statistical tests on maps of tissue change in both longitudinal and inter subject/group studies.

We also proposed a new algorithm that implements consistent matching in an intuitive manner without introducing extra penalty functions/parameters. Furthermore, the proposed algorithm provides a general recipe for inverting body forces back and forth between the forward and backward directions, and thus is applicable to any image registration schemes that compute displacement fields using incremental updating. We tested the proposed algorithm using longitudinal MRI images in a case of semantic dementia and demonstrated promising results for tracking atrophic processes in the brain.

Acknowledgements. This work was funded in part by NIH Grants R21 EB001561, R21 RR019771, and P41 RR13642. The authors would like to thank Dr. David Shattuck and Dr. Soo-Jin Lee for their valuable comments and suggestions.

\section{References}

1. P. M. Thompson and A. W. Toga, "A framework for computational anatomy," Computing and Visualization in Science, vol. 5, pp. 13-34, 2002.

2. U. Grenander and M. I. Miller, "Computational anatomy: An emerging discipline," Quarterly of Applied Mathematics, vol. 56, pp. 617-694, 1998.

3. R. P. Woods, J. C. Mazziotta, and S. R. Cherry, "MRI-PET registration with automated algorithm," J. Comput. Assist. Tomogr., vol. 17, no. 4, pp. 536-546, 1993.

4. P. Cachier and D. Rey. Symmetrization of the Non-Rigid Registration Problem using Inversion-Invariant Energies: Application to Multiple Sclerosis. In MICCAI'00, Lecture Notes in Computer Science, vol. 1935, pp 472-481, Pittsburgh, Pennsylvania, USA, October 200.

5. D. Rey, G. Subsol, H. Delingette, and N. Ayache. Automatic Detection and Segmentation of Evolving Processes in 3D Medical Images: Application to Multiple Sclerosis. In IPMI'99, Lecture Notes in Computer Science, vol. 1613, pp 154-167, Visegrád, Hungary, June 1999.

6. G. E. Christensen and H. J. Johnson, "Consistent image registration," IEEE Transactions on Image Processing, vol. 20, pp. 568-582, 2001.

7. J. Ashburner, J. Anderson, and K. Friston, "High-dimensional image registration using symmetric priors," NeuroImage, vol. 9, pp. 619-628, 1999.

8. W.M. Wells, P. Viola, H. Atsumi, S. Nakajima, and R. Kikinis, " Multi-modal volume registration by maximization of mutual information” Med. Image Anal. vol. 1(1), pp. 35-51, 1996. 